\title{
Subacromial impingement syndrome - What does this mean to and for the patient? A qualitative study.
}

\section{BACKGROUND}

Subacromial impingement syndrome (SIS) is a widely used clinical term to describe a subgroup of patients complaining of shoulder pain [1]. SIS is considered by many to be the most common of the musculoskeletal conditions affecting the shoulder, as reflected in the frequent use of the term in the published literature [2]. It is based on a hypothesis that acromial irritation leads to external abrasion of the bursa and rotator cuff [3]. However, the validity of the acromial impingement model has been challenged from both a theoretical and practical perspective [3] with some authors suggesting that such a diagnostic term promotes a viewpoint that adversely affects the science and the evolution of patient treatment $[4 ; 5]$.

The challenge to the theory of acromial irritation is based around the findings that operative outcomes post-acromioplasty are equivalent to those post-bursectomy [6]; that $71 \%$ of patients post-acromioplasty go on to develop a rotator cuff tear [7]; and that the majority of partial-thickness rotator cuff tears are found on the articular side, rather than the bursal side [8]. These findings shed doubt upon the validity of the biomedical explanation of SIS and highlight the considerable uncertainty in this area.

Further to this challenge, it has been hypothesised that SIS as a term might also be unhelpful to the patient and promote fear avoidance due to the negative connotations associated with tissue abrasion secondary to movement [5]. In the context of such diagnostic uncertainty reliance on a solely biomedical diagnosis might be unhelpful and confusing. It has been recognised elsewhere that the use of biomechanical models and the language clinician's use can increase patient fear, anxiety and stress that, in turn, negatively impacts on their clinical outcomes [9]. A wide range of factors influence patient beliefs about what is causing their problem; however, it is from healthcare professionals that patients receive the strongest influence [10]. Hence, the language and terminology we use and the way patients interpret this might be an important barrier or facilitator to good clinical outcomes. This is particularly relevant in the context of shoulder pain where 40 to $50 \%$ of people complaining of a new episode of shoulder pain continue to experience problems six to 12 months after onset [11]. Furthermore, one study identified that patients expectations of whether physiotherapy would be successful was the strongest predictor of future surgical intervention in people with shoulder pain and associated rotator cuff tear [12]. Thus, if the patient perceives their problem as one not amenable to physiotherapy, for example an osteophyte (bone spur) impinging on their rotator cuff, the opportunity to achieve a satisfactory outcome might be compromised. This is particularly unhelpful in the context of comparable outcomes between conservative care and surgery to decompress the subacromial space [13] and lack of demonstrable superiority of surgery to repair the rotator cuff [14].

Despite these concerns, there is limited understanding of how patients diagnosed with SIS understand their problem and how this might impact on their perception of physiotherapy. Hence, the aim of this qualitative study was to explore these specific factors. 


\section{METHODS}

Ethical approval was provided by $x x x x x x x x x x x x x x-x x x x x x$ Research Ethics Committee (Ref: $x x / x x / x x x x)$.

\section{Setting}

One NHS Physiotherapy department in South Yorkshire, England.

\section{Participants}

A purposive sample of patients referred to the outpatient physiotherapy department from the orthopaedic shoulder clinic, comprising consultant surgeon's and registrar's, was recruited. Patients were eligible for inclusion if they were over the age of 16 , had a diagnosis of 'subacromial impingement' and were both willing and able to consent.

On screening of the referrals and identification of a potential participant by the lead author, a patient information sheet and covering letter was posted to the potential participant detailing that they would be contacted by phone to discuss their involvement in the study. During the subsequent phone call, where the study was discussed, if the participant indicated that they would be interested in further involvement a mutually convenient time to undertake a 1:1 interview was scheduled in the Physiotherapy department where they were attending for their rehabilitation. Prior to the interview written informed consent was gained.

\section{$\underline{\text { Data collection }}$}

Interviews were conducted using a semi-structured topic guide (Appendix 1), recorded using a digital dictaphone and transcribed verbatim. The lead author, a male, conducted all interviews under the guidance and supervision of the second author who has received formal training in qualitative research methods and has previously conducted and reported qualitative research in relation to this clinical area. Participants were aware that he was also a physiotherapist by background, but would be not directly involved in their rehabilitation. Data collection continued until it was felt that no new information was arising from the interviews; two further participants were recruited at this stage to confirm this.

\section{Data analysis}

The data was analysed using the Framework method [15], which has been developed specifically for applied research in which the objectives of the study are set a priori [16]. Analysis began with familiarisation of the data, which further informed the development of a thematic framework. This framework then formed the basis upon which the key issues and themes were developed and by which the data was examined. Subsequently the data was indexed according to this framework before a charting process was completed; this is where the data was organised according to the defined thematic framework. These charts were then used to define the concepts and find associations to provide explanations for the findings $[15,16]$. Following familiarisation, both authors agreed on the initial thematic framework. Indexing was initiated by the lead author, prior to charting, and subsequently developed and verified by the second author. Initial discrepancies in interpretation were resolved through discussion without the need for further arbitration and the thematic framework evolved as a basis on which the mutually agreed narrative was developed. 


\section{RESULTS}

Nine participants diagnosed with SIS were approached and all were recruited and interviewed; three prior to their initial physiotherapy appointment and six after. Four of the patients were male $(44.4 \%)$ and the mean age was 56 years and six months.

The length of the interviews was variable but typically lasted approximately ten minutes. Three main themes were generated: (1) The diagnostic experience, (2) Understanding of the problem, (3) Expectation of the treatment required; with one sub-theme: (3b) Barriers to engagement with physiotherapy.

\section{The Diagnostic Experience}

The majority of patients (7/9) recruited to this study reported similar diagnostic experiences whereby there was apparent initial uncertainty on the part of the GP or orthopaedic team but this uncertainty was countered, in the minds of the patients, by referral for a scan, which was assumed to be capable of providing the necessary diagnostic answers:

"I went to see my GP who said that he thought I may have a tear in the ligament or tendon or whatever and sent me for a shoulder x-ray and an ultrasound scan". (ID 2)

"GP examined my shoulder, made me do various movements and sent me off for an x-ray". (ID 1)

"I had some $x$-rays done, and in fact prior to that when meeting with him, the Consultant seemed to think it was a frozen shoulder but it wasn't, he said it was a bit like a frozen shoulder but it's not, he seemed a little bit puzzled by it. So, I had these $x$-rays, the $x$-ray came back and he came into see me and he said, I know what it is". (ID 3)

"The Consultant thought that it might have been something to do with my biceps muscles, you know, where they attach so he sent me to have a scan, an ultrasound scan". (ID 4)

For all the patients (9/9), it was apparent that clinical reasoning was grounded within a biomedical model and where scans were performed they were seen as capable of providing definitive answers. Although open to question, such reasoning within a biomedical framework appeared to align well with the beliefs, values and expectations of the patients:

"... and while the Dr, she were scanning it she told me that the lining on one of the ligaments or tendons was swollen... the ultrasound scan, she knew what she were doing, she knew what were wrong, so yeah, l'd recommend that". (ID 4)

(Upon referral to the Consultant) "She was like the most helpfulest person out of everybody because I've had an x-ray and I've been booked in for an ultrasound scan". (ID 6)

So, in this context a predominantly biomedical based approach to clinical reasoning is the dominant approach where information garnered from a scan is regarded as authoritative. Such an approach appears to satisfy and align well with the expectations of the patients. As would be expected, it is evident that this approach to diagnosis impacts on how the patient begins to conceptualise and understand their problem with subsequent implications for physiotherapy management.

Understanding of the Problem 
All of the patients (9/9) in this study described their understanding of the problem in a consistent way aligned with a biomedical understanding of the model of SIS:

"It is the tendon being caught by this piece of bone and wearing it away.". (ID 3)

"According to my doctor, I had a scan and I've got two extra bones... They did say there is inflammation and there's something that's rubbing and then every time it rubs that's what causing me to have pain... He (Consultant) just said it was all swollen up and that there's something that when it's swollen up that's what's causing me the pain and that's as much as I know". (ID 5)

"He said there was some impingement that could have been inflammation from irritation in the shoulder...things stopping my arm actually moving the way it should...I could just picture something just in the joint that got in the way at a certain point and was making it difficult for the socket to move in the way it should be moving". (ID 9)

Although there was consistency in the way the patients described their understanding of the problem when they recalled the information relayed during the consultation, it was apparent that not all patients (2/9) recounted the same level of detail:

"They might have explained something at the time but I can't remember". (ID 4)

"If there was inflammation in the joint, I'll be having an injection and some tablets and if it were a different one there would be another course of action but I can't remember what that was." (ID 6)

Despite questions over the validity of the biomedical model and the subacromial impingement model, as initially described, it seems that these views continue to be impressed on patients with this subgroup of shoulder pain. Although not all patients seem to recall the specifics of the diagnostic detail, it is apparent that such understanding, impressed on the patient by the attending clinician, has a direct impact on how they view the effectiveness of treatment interventions and future treatment pathway.

\section{Expectation of the Required Treatment}

As might be expected, it was evident that patients expected treatment that was aligned to their understanding of the problem; the treatment needed to match the cause for it to be deemed worthwhile. Most (7/9) participants reported this directly:

"(How are we going to get this pain to go away?) By removing this piece of bone." (ID 3)

Despite this, there was recognition that conservative treatment options should be trialled first with the aim of avoiding any surgical intervention:

"He (Consultant) gave me an injection in to the shoulder and said that if the injection and the physiotherapy didn't work then he would have to consider surgery." (ID 1)

"He (Consultant) was on about sending me for a scan but then he just said he'll try this injection first and see how I get on and then physiotherapy and then go back and see how I'm doing." (ID 5)

"(An Injection)... an option to try first, that if that works then it would save any more drastic treatment and so it was a progression thing to see how it would help." (ID 9) 
Despite understanding their shoulder problem in what might be seen as an unhelpful way from the perspective of conservative management, it was apparent that some patients (3/9) recognised the risks associated with surgery and hence were open to the concept of stepped-care beginning with conservative measures:

"Well I always think of surgery as the means of last resort, try everything else first.." (ID 1)

"I'd rather not have surgery if I can avoid it; I'd do anything other than surgery." (ID 2)

"I mean obviously you do look up, don't you, on your internet as soon as you get out of the [laughter], and I think basically, yes, it did say there was...options: obviously two which I've already tried, the painkillers and the steroid; and then obviously the final one is open surgery". (ID 7)

However, some contradiction to this narrative was apparent. Even though the patients appeared to appreciate the rationale underpinning stepped-care from a risk perspective, doubts remained about the effectiveness of such an approach with regards to clinical effectiveness:

"(Try everything else first) and if that doesn't offer a long term solution than surgery may be the only method of gaining the function back on either a permanent or longer basis". (ID 1)

"I don't want to get to a position where it's okay, I go back to work and it blows up again and I'm back to square one. I want it sorted you know. I said to the Physiotherapist you know, I think the only way that is going to permanently cure this is through that surgery, you know". (ID 3)

\section{Barriers to engagement with physiotherapy}

Further, linked with patient understanding and expectations, in some circumstances there appears to be direct implications for physiotherapy management in terms of how the participants perceived the potential effectiveness of physiotherapy, with some (5/9) questioning the appropriateness:

"If there is a physical lump or something there, all the Physiotherapy in the world isn't going to resolve that if that doesn't disappear. He said that it was quite likely in my case that I might well need some keyhole surgery...". (ID 1)

"There is a possibility, I think, I think what the Consultant was meaning was that I could have some Physio to get the inflammation down, get the swelling down, get the muscles stronger but it's not permanent, it's not going to be a permanent fix, this piece of bone is still there. I can't imagine how any amount of physio is going to shift this piece of bone which is in my shoulder." (ID 3)

It was apparent that the message delivered by the attending clinician influences subsequent expectations of what Physiotherapy can achieve:

"He said we can do keyhole surgery on it and it'll be fine. Meanwhile, he said I want you to try a couple of months of physio first before we commit to that, which puzzles me to be honest because I thought well you know, if this piece of bone is not a big job just go in there, get rid of this bit of bone, fine. So I was a bit nonplussed when I came to physio, I thought well you know, I felt a bit sorry for the guy to be honest". (ID 3)

"..Why do I have to waste your time being here?". (ID 6) 
This conflict appears to be grounded in an uncritical view of the biomedical approach to diagnosis and also a lack of understanding to what physiotherapy might help a patient to achieve:

"The Consultant can offer me the way forward, Physiotherapy can't really. They don't understand what is going off inside it, do they??... I don't even understand Physiotherapy to be fair" (ID 6)

"I couldn't see how Physiotherapy would help with a tear...I'd be worried that I was doing even more damage". (ID 2)

\section{DISCUSSION}

In this study patients referred to physiotherapy by an orthopaedic surgeon/ registrar from a specialist shoulder clinic described a diagnostic experience grounded in the biomedical model where early diagnostic uncertainty was apparent but imaging was viewed as being capable of providing authoritative guidance. In response to their clinical encounter, patients, on the whole, developed their understanding of their shoulder problem in line with ta biomedical and/ or subacromial impingement model where tissues in the shoulder were seen to be compressed, abrased or inflamed while the shoulder was moved. Such an understanding had an impact on what patients perceived to be the most effective treatment pathway. Furthermore, such an understanding of the problem and the ensuing belief about the most effective treatment, had implications for how physiotherapy was viewed which could potentially serve as a barrier to engagement and successful clinical outcome.

As far as we are aware, this is the first study to consider these diagnostic and terminology issues from the perspective of patients diagnosed with SIS. Although limited by context, these findings raise questions for clinicians, including physiotherapists and surgeons, involved in the management of these shoulder conditions. In a situation where patients expectations of whether physiotherapy would be successful are strongly associated with success [12], it seems sensible to suggest that it is important that patients commence physiotherapy with realistic but optimistic expectations rather than pessimistic expectations. It seems based on the findings of this study that, among other things, pessimistic expectations can be developed secondary to developing an understanding of their problem that was not amenable to physiotherapy, for example perceiving that their shoulder pain is attributable to a rotator cuff tear.

Despite recognition that structural pathology is not well associated with pain [5], is not predictive of treatment outcome [17] and does not change as patients report reduced pain and improved function [18], this model of understanding continues to underpin much of current clinical practice. The narrative here highlights how understanding based on such a biomedical model of pain might be unhelpful to both patients and physiotherapists. There is now evidence suggesting that psychosocial factors contribute to the persistence of shoulder pain [19], and the relevance of central pain mechanisms has been recognised [20]. Within the context of this study, it did not seem that such factors were given credence by the attending clinician, at least from the perspective of the patient, and thus were not apparent in the patients understanding of their problem. Furthermore, patients within this study demonstrated an underlying feeling of vulnerability and subsequent avoidance of certain activities through fear of further tissue abrasion secondary to movement, highlighting how language aligned to the theory of acromial irritation can not only impact upon clinical outcomes and subsequent management but also contribute to chronicity of symptoms.

On this backdrop it is important to recognise that associated with such diagnostic uncertainty is an uncertainty about the most appropriate terminology to use and model to explain the pain that patients complain of [5]. A range of diagnostic terms are currently used for patients complaining of shoulder pain exacerbated with movement or load but where movement is largely maintained; 
these terms include rotator cuff related shoulder pain, subacromial pain syndrome and rotator cuff tendinopathy. Although these terms are imperfect and likely to evolve over time, based on this narrative, we are suggesting that clinicians should consider the impact of the language they use with patients in terms of how it might influence engagement and treatment success along the entire treatment pathway. In this context, using the term SIS grounded in the biomedical model potentially contributes to pessimistic expectations of physiotherapy, which might compromise outcome. In the context of research evidence suggesting at least comparable clinical outcomes between surgery and physiotherapy for people with this subgroup of shoulder pain [13], but at substantially less cost, this is a concern because it might result in unnecessary referral for surgical intervention at significantly greater cost.

Recognition of these issues is the first step to effecting change. We suggest that clinicians from similar and different backgrounds involved in the management of patients with musculoskeletal pain aim to engage in constructive discussion about advice given and language used; this appears most paramount for clinicians working in similar localities where the potential for patients to receive conflicting messages is more likely. Secondly, we now have a body of evidence that challenges some of the assumptions underpinning the biomedical and subacromial impingement model. It is recommended that this research evidence is used to challenge the unhelpful beliefs of clinical colleagues and patients through various means, including patient friendly infographics highlighting the aforementioned issues with one aim to do no harm with the language and terms we use.

\section{$\underline{\text { Limitations }}$}

This study was conducted in one NHS hospital with patients referred from one specialist shoulder clinic. Although most would now not judge qualitative research from the perspective of its capacity to generate data regarded as being generalisable, such a context might hamper the transferability of the findings. Furthermore, the study was conducted by two researchers who are physiotherapists by background which might give rise to questions around the credibility and confirmability of the findings. However, a transparent method of data analysis was adopted and it was apparent that participants felt able to discuss the role of physiotherapy in the context of their current diagnosis in both a positive and negative light which might suggest that the role of the researcher did not have a negative influence.

\section{CONCLUSION}

The findings from this study suggest that diagnosis of shoulder pain remains grounded in a biomedical model where identification of the structure at fault is a priority and diagnostic imaging forms a central component to this decision making. Understanding and explaining pain using the subacromial impingement model seems acceptable to patients but might have significant implications for engagement with and success of physiotherapy. It is suggested that clinicians should be mindful of the terminology they use and consider its impact on the patient's treatment pathway with the aim of doing no harm with the language used.

\section{Funding: Nil}

Conflict of Interest: None. 


\section{REFERENCES}

[1] Littlewood C, Ashton J, Chance-Larsen K, May S, Sturrock B. Exercise for rotator cuff tendinopathy : a systematic review. Physiotherapy 2012;98:101-9.

[2] Littlewood C, May S, Walters S. A review of systematic reviews of the effectiveness of conservative interventions for rotator cuff tendinopathy. Shoulder Elb 2013;5:15167. doi:10.1111/sae.12009.

[3] Lewis J. Subacromial impingement syndrome: A musculoskeletal condition or a clinical illusion? Physical Therapy Reviews 2011;16:388-298.

[4] McFarland E, Maffulli N, Del Buono A, Murrel G, Garzon-Muvdi J, Peterson S. Impingment is not impingement: the case for calling it "Rotator Cuff Disease". Muscles Ligaments Tendons J 2013;3;196-200.

[5] Littlewood C, Malliaras P, Bateman M, Stace R, May S, Walters S. The central nervous system - An additional consideration in "rotator cuff tendinopathy" and a potential basis for understanding response to loaded therapeutic exercise. Man Ther 387 2013;18:468-72.

[6] Donigan J, Wolf B. Arthroscopic subacromial decompression: acromioplasty versus bursectomy alone - does it really matter? A systematic review. lowa Orthop J 2011; 31:121-126.

[7] Hyvonen P, Lohi S, Jalovaara P. Open acromioplasty does not prevent the progression of an impingement syndrome to a tear. Nine year follow up of 96 cases. J Bone Jt Surg 1998; 80:813-816.

[8] Uhthoff H, Sano H. Pathology of failure of the rotator cuff tendon. Orthopedic Clinics of North America 1997; 28: 31-41.

[9] Coudeyre E, Rannou F, Tubach F, Baron G, Coriat F, Brin 396 S, Revel M, Poiraudeau S. General practitioners' fear-avoidance beliefs influence their management of patients with low back pain. Pain 2006; 124:330-337.

10] Darlow B, Dowell A, Baxter G, Perry M, Dean S. The Enduring Impact of What Clinicians Say to People With Low Back Pain. Ann Fam Med 2013; 11:527-534.

[11] Ottenheijm R, Joore M, Walenkamp G, Weijers R, Winkens B, Cals J, et al. The Maastricht Ultrasound Shoulder pain trial (MUST): ultrasound imaging as a diagnostic triage tool to improve management of patients with non-chronic shoulder pain in primary care. BMC Musculoskelet Disord 2011;12.

[12] Dunn W, Kuhn J, Sanders R, An Q, Baumgarten K, Bishop J. 2013 Neer Award: predictors of failure of nonoperative treatment of chronic, symptomatic, full-thickness rotator cuff tears. J Shoulder Elb Surg 2016;25:1303-11. 
[13] Ketola S, Lehtinen J, Arnala I, Nissinen M, Westenius H, Sintonen H, et al. Does arthroscopic acromioplasty provide any additional value in the treatment of shoulder impingement syndrome? A two year randomised controlled trial. J Bone Jt Surg 2009;91:1326-34.

[14] Sealey P, Lewis J, Sealey P. Rotator cuff tears: is non-surgical management effective ? Rotator cuff tears : is non-surgical management effective ? Phys Ther Rev 2016;3196:0. doi:10.1080/10833196.2016.1271504.

[15] Lacey A, and Luff D. Trent focus for research \& development in primary health care: an introduction to qualitative analysis. The NIHR RDS for the East Midlands, Yorkshire \& the Humber; 2007

[16] Pope C, Ziebland S, Mays N. Analysis qualitative data. BMJ 2000; doi: https://doi.org/10.1136/bmj.320.7227.114

[17] Littlewood C, May S, Walters S. Epidemiology of rotator cuff tendinopathy: a systematic review. Shoulder Elb 2013;5:256-65.

[18] Drew B, Smith T, Littlewood C, Sturrock B. Do structural changes (eg, collagen/matrix) explain the response to therapeutic exercises in tendinopathy? A systematic review.

Br J Sports Med 2014; 48(12):966-72.

[19] Chester R, Jerosch-Herold C, Lewis J, Shepstone L. Psychological factors are associated with the outcome of physiotherapy for people with shoulder pain: a multicentre longitudinal cohort study. Br J Sports Med 2016.

[20] Gwilym W, Oag H, Tracey I, Carr A. Evidence that central sensitisation is present in patients with shoulder impingement syndrome and influences the outcomes after surgery. $J$ Bone Joint Surg 2011; 93-B:498-502. 


\section{Appendix 1}

\section{Topic Guide}

1) Thank you for agreeing to participate in our study, would you like to start off by telling us your story? How did we get to where we are today?

2) What do you understand to be the cause of your shoulder pain?

3) If you were to picture your shoulder in your mind, what do you see?

4) How do you think this pain can be resolved?

5) How do you think Physiotherapy will help you? 